\title{
Symptoms of Temporomandibular Dysfunction in Patients Submitted to Bucco-Dental Surgery
}

\author{
Thaís Cristina Araújo Moreira', Lidia Audrey Rocha Valadas²*, Antônio Sérgio Guimarães \\ 'São Leopoldo Mandic School of Dentistry, Fortaleza, CE, BRAZIL. \\ ${ }^{2}$ Federal University of Ceara, Fortaleza- CE, BRAZIL.
}

\section{ABSTRACT}

Objectives: To verify the symptoms of temporomandibular disorders (TMD) in a sample of patients who made dental surgery, before and after the procedure. Methods: This is a clinical, longitudinal and quantitative study. The study population consisted of patients of both genders, aged between 16 to 60 years, selected in the surgery service. The participants answered two questionnaires to evaluate the presence of TMD symptoms, the TMD-Pain Screener questionnaire and the questionnaire of the European Academy of Craniomandibular Disorders (EACD). Results: 110 patients were evaluated, the most female $(p<0.05)$. When questioned about which surgery was performed, the highest prevalence was of impacted third molars $(37.3 \%)$, followed by third erupted molars $(27.2 \%)$ and third molars included $(18.2 \%)$. When evaluating the results of the TMD-Pain Screener questionnaire, there was statistical significance for all questions both in the pre- and post-operative periods. Regarding the EACD questionnaire there was no statistical significance for pain. When comparing the questionnaires at different times, there was no statistical difference in the EACD questionnaire and TMD-Pain Screener, only in relation to mouth opening and laterality movements $(p=0.04)$. Patients with and withoutTMD
\end{abstract}

symptoms showed no statistical significance for any of the questionnaires. Conclusion: It was concluded that there was a high prevalence of painful TMD symptoms in patients who sought dental surgery. After the surgical procedure, there was a significant decrease in mandibular pain in the opening, protrusion and laterality movements. Thus, dental surgeries performed under adequate operative conditions do not affect TMD symptoms in patients.

Key words: Temporomandibular Joint, Facial Pain, Signs and Symptoms, Ambulatorial Surgery Procedures, Oral Surgery.

Correspondence

Dr. Lidia Audrey Rocha Valadas,

Federal University of Ceara, Fortaleza- CE, BRAZIL.

Phone: +558533668000

Email: lidiavaladas@hotmail.com

DOI: 10.5530/jyp.2020.12.28

\section{INTRODUCTION}

The temporomandibular joint (TMJ) is one of the main joints of the human body. ${ }^{1}$ When the physiology of the system that makes up the TMJ is altered, temporomandibular disorder (TMD) occurs. TMD has two classifications: they can be of articular origin, where the signs and symptoms are related to TMJ or of muscular origin, where the signs and symptoms are related to the stomatognathic musculature. Thus, TMD is related to structural, neuromuscular, occlusal, psychological factors, habits for functional and traumatic or degenerative TMJ injuries. ${ }^{2}$

The main signs and symptoms that characterize TMD are joint noises and crackles, tinnitus, headache, limitations or deviations in jaw movements, myalgia, earache, tenderness, pain in the stomatognathic system and, in more serious situations, dislocations. ${ }^{3}$ TMD signs and symptoms, such as trismus, otalgia and pain in the chewing muscles, may be present in patients with indication for removal of third molars. ${ }^{4}$ Experimental and clinical studies indicate that changes in muscle movements and exacerbation of pain may occur after surgery. Therefore, it is important to consider the possible changes that may interfere with the success of a surgical procedure..$^{5-7}$

The verification of these signs and symptoms can be performed by questionnaires such as DC / TMD, which are currently the most widely used standardized diagnostic criteria for TMD, with great reliability and validity, covering muscle disorders, disc displacement, arthralgia, osteoarthritis and osteoarthritis. In addition, it assesses psychosocial dysfunction and disability both related to pain. ${ }^{8}$
Juhl et al. ${ }^{9}$ described that any surgical procedure, even a small one, can result in injury leading to an impact on quality of life and risk of chronic pain. According to Huang et al. ${ }^{5}$ surgery to remove third molars is a risk factor for developing painful TMJ symptoms, as it is a type of procedure that can lead to trauma in the temporomandibular region due to excessive mouth opening and for prolonged periods and injuries to muscles and joints can occur.

Huang and Rue $^{6}$ evaluated the possible association between extraction of third molars and TMD. The authors followed for 5 years patients initially aged 15 years. The authors investigated the development of TMD after extraction of the third molars. There was a $60 \%$ increase in the risk of TMD symptoms in the sample after having their third molars removed.

Sharma and Narain ${ }^{10}$ report that the roots of the lower molars can compress and damage the lower lveolar nerve, usually in cases in which they trap the mandibular canal, lead to painful conditions that can progress causing orofacial pain. In such cases, surgical interventions are indicated because permanent nerve damage may still occur. The compression of the blood supply by the roots of impacted third molars results in increased vascular permeability, edema and ischemia. Although the traumatic and ischemic injury has recovery in around 8 weeks in $85 \%-94 \%$ of cases, prolonged compression can cause degeneration of the fibers.

The aim of this study was to check TMD symptoms in a sample of patients who underwent oral-dental surgery. In addition, to compare the pre 
and post-operative moments in order to observe whether the surgical procedure influenced the prevalence of TMD symptoms in the sample.

\section{MATERIALS AND METHODS}

\section{Type of study, location and period of research}

This is a clinical, cohort and prospective study. The study was approved by the research ethics committee under number 2.541.250.

The study population consisted of patients of both genders, between 16 and 60 years old, who sought dental care at the University Hospital and Instituto LatoSenso, both in Piauí-Brazil with an indication for oral or dental surgery.

\section{Inclusion and exclusion criteria}

The following inclusion criteria were considered: Patients of both genders, between 16 and 60 years old; Patients with indication for oral-dental surgery, such as third molars, multiple extractions, pre-prosthetic surgery, biopsy and odontoma. The exclusion criteria were: Patient without indication for surgery; Patients not able to answer the questionnaire; Patients who did not respond to the IC; Patients who did not return for postoperative.

\section{Data collection instrument}

In the pre- and postoperative period ( 15 days after surgery) the participants answered two validated questionnaires to assess the presence of TMD symptoms, composed of questions belonging to questionnaire "TMD-Pain Screener" and the questionnaire of the European Academy of Disorders mandibular cranium.

\section{Statistical analysis}

The data were tabulated in a standard Microsoft Office Excel 2010 spreadsheet and exported to the Statistical Package for the Social Sciences (SPSS) software version 20.0 for Windows, in which the analyzes were performed adopting 95\% confidence. For comparison, non-parametric tests were used according to the Kolmogorov-Smirnov normality test.

\section{RESULTS}

110 patients were evaluated, of which $71 \%(n=78)$ were female and $29 \%$ $(n=32)$ male, with statistical difference between groups $(p<0.05)$.

\section{Surgery}

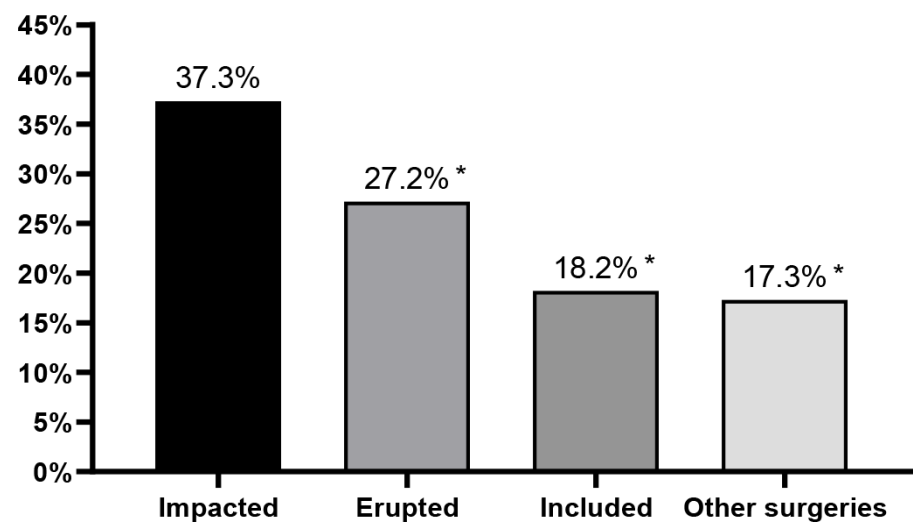

Graph 1: Types of surgeries performed in the sample.

Subtitle: Gray columns explaining the types of surgery: impacted $(37.3 \%$; $n=41)$, erupted $(27.2 \% ; n=30)$, included $(18.2 \% ; n=20)$ and other surgeries $(17,3 \% ; \mathrm{n}=19) .{ }^{*} p<0.05$ vs. impacted, ANOVA, followed by the Dunns method. @ $p<0.05$ vs. erupted, ANOVA, followed by the Dunns method. Source: Own authorship.
When asked about which surgery was performed, the highest prevalence was of patients with impacted third molars $(37.3 \% ; n=41)$, followed by erupted third molars $(27.2 \% ; n=30)$ and included third molars $(18.2 \%$; $n=20)$ (Graph1).

Table 1 compares the TMD-Pain Screener questionnaire at different times. There was statistical significance regarding the question about the presence of pain when opening the mouth or moving the mandible forward and to the sides $(p=0.04)$.

Table 2 compares the European Academy of Craniomandibular Disorders questionnaire at different times. There was no statistical difference when analyzing the different times.

Table 3 compares the responses to the TMD-Pain Screener questionnaire, of individuals who presented TMD symptoms preoperatively with those who did not present us with different times. There was no statistical difference when analyzing the different groups at both times.

Table 4 compares the responses of the EACD questionnaire, of the individuals who had TMD symptoms in the preoperative period with those who did not present at different times. There was no statistical difference when analyzing the different groups at both times.

Table 1: Comparison of the TMD - Pain Screener questionnaire in the pre and postoperative period.

\begin{tabular}{|c|c|c|c|}
\hline & Before & After & \\
\hline Questions & n (\%) & $\mathrm{n}(\%)$ & $P$ \\
\hline \multirow{2}{*}{\multicolumn{4}{|c|}{$\begin{array}{c}\text { 1. In the past } 30 \text { days, did you experience } \\
\text { pain in your jaw, temporal area on both } \\
\text { sides? }\end{array}$}} \\
\hline & & $52(47.5 \%)$ & 0.47 \\
\hline Painless & $53(48 \%)$ & $52(47.5 \%)$ & \\
\hline Altered pain & $3(3 \%)$ & $6(5.4 \%)$ & \\
\hline Present pain & & & \\
\hline \multicolumn{4}{|l|}{$\begin{array}{l}\text { 2. In the past } 30 \text { days, did you experience } \\
\text { pain or a jaw lock upon waking up? }\end{array}$} \\
\hline No & $23(21 \%)$ & $22(20 \%)$ & 0.84 \\
\hline Yes & $87(79 \%)$ & $88(80 \%)$ & \\
\hline \multicolumn{4}{|l|}{$\begin{array}{l}\text { 3. In the last } 30 \text { days, the following } \\
\text { activities had some change (improvement } \\
\text { and worsening) related to pain in the jaw, } \\
\text { temporal area on both sides: }\end{array}$} \\
\hline \multicolumn{4}{|l|}{ A) Hard foods or continuous chewing? } \\
\hline No & $20(18 \%)$ & $26(23.6 \%)$ & 0.21 \\
\hline Yes & $90(82 \%)$ & $84(76.4 \%)$ & \\
\hline \multicolumn{4}{|l|}{$\begin{array}{l}\text { B) Open your mouth or move your jaw } \\
\text { forward and sideways? }\end{array}$} \\
\hline No & & & \\
\hline Yes & $20(18 \%)$ & $30(27.3 \%)$ & 0.04 \\
\hline $\begin{array}{c}\text { C) Mandibular habits, such as keeping } \\
\text { your teeth together, grinding or chewing } \\
\text { gum? }\end{array}$ & $90(82 \%)$ & $80(72.7 \%)$ & \\
\hline No & $22(20 \%)$ & $22(20 \%)$ & 1.00 \\
\hline Yes & $88(80 \%)$ & $88(80 \%)$ & \\
\hline \multicolumn{4}{|l|}{$\begin{array}{l}\text { D) Other activities like talking, kissing and } \\
\text { yawning? }\end{array}$} \\
\hline No & $20(18 \%)$ & $26(23.6 \%)$ & 0.21 \\
\hline Yes & $90(82 \%)$ & $84(76.4 \%)$ & \\
\hline
\end{tabular}

Subtitle: Before $=$ preoperative, After $=$ postoperative. $n=$ number of subjects, $\%=$ relative frequency in percentage. $\mathrm{P}=P$ value. ${ }^{\star}$ Paired Wilcoxon test. Source: Own authorship. 
Table 2: Application of the European Academy of Cranio Mandibular Disorders Questionnaire in the pre and post-operative period.

\begin{tabular}{cccc}
\hline & Before & After & \\
\hline Questions & $\mathbf{n}(\%)$ & $\mathbf{n}(\%)$ & $P$ \\
\hline $\begin{array}{c}\text { 1. Do you have pain when you open } \\
\text { your mouth widely or chew once a } \\
\text { week or more? }\end{array}$ & & & \\
Yes & $24(22 \%)$ & $25(22.7 \%)$ & 0.85 \\
No & $86(78 \%)$ & $85(77.3 \%)$ & \\
2. Do you have pain in your temples, & & & \\
face, temporomandibular joints or & & & \\
jaws, once a week or more? & & & \\
Yes & $46(42 \%)$ & $49(44.5 \%)$ & 0.59 \\
No & $64(58 \%)$ & $61(55.5 \%)$ & \\
3. Have you registered your jaw lately & & & \\
or are you unable to open it widely? & & & \\
Yes & $9(8 \%)$ & $14(13 \%)$ & 0.24 \\
No & $101(92 \%)$ & $96(87 \%)$ & \\
4. Do you often have headaches, once a & & & \\
week or more? & & & \\
Yes & & & \\
No & $61(44,5 \%)$ & $50(45.5 \%)$ & 0.85 \\
\hline
\end{tabular}

Subtitle: Before $=$ preoperative, After $=$ postoperative. $n=$ number of subjects, $\%=$ relative frequency in percentage. $\mathrm{P}=P$ value. ${ }^{*}$ Paired Wilcoxon test.

Source: Own authorship.

Table 3: Comparative analysis before (pre) and after (post) the surgical procedure in relation to the TMD-Pain Screener questionnaire.

\begin{tabular}{cccc}
\hline Pain Screener & Pre-operative & Post-operative & $P$ \\
\hline No & 0 & 0 & \\
Yes & $110(50 \%)$ & $110(50 \%)$ & $>0.05$ \\
Yes everything & $22(10 \%)$ & $17(7.8 \%)$ & \\
Up to 5 week & $88(40 \%)$ & $93(42.2 \%)$ & \\
\hline
\end{tabular}

Subtitle: Number of subjects (relative frequency in percentage). $P=P$ value, paired $t$-test.

Source: Own authorship.

Table 4: Comparative analysis before (pre) and after (post) the surgical procedure taking into account the EACD.

\begin{tabular}{cccc}
\hline EACD & Pre-operative & Post-operative & $\boldsymbol{P}$ \\
\hline No & $52(47.2 \%)$ & $49(44.6 \%)$ & $>0.05$ \\
Yes & $58(52.8 \%)$ & $61(55.4 \%)$ & \\
\hline
\end{tabular}

Subtitle: Number of subjects (relative frequency in percentage). $P=P$ value, paired $t$-test.

Source: Own authorship.

\section{DISCUSSION}

In the present study, it was found that the most of patients in the total sample were female $(70.9 \%)$ with a mean age of 28 years. However, considering the age group up to 30 years of age, the male gender prevailed (83.9\%).

There are few articles that relate orofacial pain and oral or dental surgery, where those found in the literature are usually related to trigeminal neuralgia. ${ }^{11}$ Studies report that surgical procedures, even those considered small, can contribute to the onset or intensification of chronic orofacial pain, including TMD symptoms., ${ }^{9,12,13}$

Studies report that when performed unilareral extractions, the masseter and medial pterygoid muscles, with mandibular movement functions, show adaptive neuromuscular changes that can culminate in TMD symptoms such as stress and muscle tension, but that an adaptation of these structures can be observed around 60 days after surgical procedures. ${ }^{7}$ In this study, most extractions of third molars were performed $(82.7 \%)$. Other surgeries, such as pre-prosthetic surgeries, removal of odontomas, multiple extractions (over 5 elements), together correspond to a percentage of $17.3 \%$.

In the present study, the most performed extractions were those of impacted third molars (37.3\%), followed by erupted third molars (27.2\%) and inclusions (18.2\%). The literature points out that the third molars are related to several disorders in the orofacial region, such as pericoronaritis, dental crowding, difficulty eating, headaches, trismus, otalgia, muscle sensitivity, among other problems.

In a study that assessed the prevalence of patients with pain and TMD and indication for removal of the third molar, a total of $13.3 \%$ had signs and symptoms related to TMD, thus suggesting that signs of TMJ disorders may be common in these patients. ${ }^{4}$ In this study, all patients reported the presence of pain according to the TMD-Pain Screener.

Huang et al. ${ }^{2}$ evaluated the temporal relationship in patients with a report of trauma in the removal of the third molar before the onset of TMD. In this study, factors such as trauma, tightening, removal of the third molar, somatization were identified as risk factors for the onset of myofascial pain. In the present study, all patients reported the presence of pain according to the TMD-Pain Screener $(p<0.05)$. In addition, all questions on the TMD-Pain Screener also showed significance $(p<0.05)$.

Studies associate complications such as TMD after extraction of third molars due to the need for the patient to have to open the mouth for a long period and also due to trauma, especially in the jaw. However, many studies that investigate possible complications after the removal of third molars ignore TMD, paying more attention to immediate complications such as bleeding, pain at the surgery site, paraesthesia, infection, inflammation, among others. ${ }^{6}$ The present research showed significant results regarding the symptoms of pain, changes in the jaw and in the activities performed for all questions asked in the postoperative period $(p<0.05)$.

Several factors must be considered during a clinical evaluation in order to discover the cause of these TMD symptoms. Trauma is known to result in injury and pain. The extraction of third molars, because it involves a wide mouth opening, a considerable force in the jaw, among other factors, can result in trauma to the TMJ and chewing muscles. However, the association of the appearance of TMD after the removal of these teeth requires further studies. ${ }^{5}$

The impact of surgery on TMD signs and symptoms is not well understood, as many of the clinical studies that evaluate this have a limited number of patients evaluated do not have a control group or standardization of age group. ${ }^{14}$ It is interesting that surgical techniques seek to reduce the load and the forces on the TMJ and the muscles and often a more conservative decision regarding the general indications for the removal of third molars. ${ }^{9}$

Another study evaluated the prevalence of TMD symptoms in a sample of patients from a buccomaxillofacial service in Pernambuco. After applying a questionnaire, it was observed that pain was present in $100 \%$ of the sample. ${ }^{15}$ Considering the EACD questionnaire for TMD signs and symptoms, the participants of this survey mainly pointed out the occurrence of frequent headaches at least once a week. In the present 
study, there was no statistical difference in the questions of the EACD questionnaire, when comparing the different moments.

Juhl et al. ${ }^{9}$ also used DCTMD to assess the association between the development of TMD signs and symptoms after surgery on third molars for a period of six months. One week later, there was an increase in pain and a reduction in the maximum opening. Muscle and TMJ pain on palpation was reported up to 6 months after surgery, these symptoms being statistically significant compared to the control group. In the present study there was no significant difference between the moments. Differences in methodologies and drug protocol may have influenced the difference in results.

\section{CONCLUSION}

Considering the applied questionnaires, it is concluded that there was a high prevalence of painful TMD symptoms in patients who sought dental surgery, mainly considering the TMD - Pain Screener questionnaire, which considers jaw locking and pain in the mandibular and temporal region of the last 30 days. After the surgical procedure, there was a significant decrease in mandibular pain in the opening, protrusion and laterality movements.

\section{ACKNOWLEDGEMENT}

The authors acknowledge São Leopoldo Mandic School of Dentistry, Hospital of Federal University of Piaui, LatoSenso Institute.

\section{CONFLICT OF INTEREST}

The authors declare no conflict of interests.

\section{ABBREVIATIONS}

DC: Diagnostic Criteria; EACD: European Academy of Craniomandibular Disorders; TMD: Temporomandibular Disorder; TMJ: Temporomandibular Joint.

\section{REFERENCES}

1. Fernandes LM, Marques LARV, Rodrigues EMN, Lotif MAL, Melo FHC Macena RHM. Profile of temporomandibular disorder patients submitted to dental and physiotherapeutic treatment at a private clinic. Rev Sul-Bras Odontol. 2014;11(3):260-4

2. Donnarumma MDC, Muzilli CA, Ferreira C, Nemr K. Disfunções Temporomandibulares: sinais, sintomas e abordagem multidisciplinar. Rev CEFAC. 2010;12(5):788-94.

3. Machado E, Grehs RA, Cunali PA. Imaginologia da articulação temporomandibular durante o tratamento ortodôntico: uma revisão sistemática. Dental Press J Orthod. 2011;16(3):54-6.

4. DeAngelis A, Chambers I, Hall G. Temporomandibular joint disorders in patients referred for third molar extraction. Aust Dent J. 2009;54(4):323-5.

5. Huang GJ, LeResche L, Critchlow CW, Martin MD, Drangsholt MT. Risk Factors for Diagnostic Subgroups of Painful Temporomandibular Disorders (TMD). J Dent Res. 2002;81(4):284-8

6. Huang GJ, RueTC. Third-molar extraction as a risk factor for temporomandibular disorder. J Am Dent Assoc. 2006:137(11):1547-54

7. Bazan E, Issa JPM, Watanabe I, Mandarim-de-Lacerda CA, DelBel EA, Iyomasa MM. Ultrastructural and biochemical changes of the medial pterygoid muscle induced by unilateral exodontia. Micron. 2008;39(5):536-43.

8. Talaat WM, Adel OI, AlBayatti S. Prevalence of temporomandibular disorders discovered incidentally during routine dental examination using the Research Diagnostic Criteria for Temporomandibular Disorders. Oral Surg. Oral Med. Oral Pathol Oral Radiol. 2018;125(3):250-9.

9. Juhl GI, Jensen TS, Norholt SE, Svensson P. Incidence of symptoms and signs of TMD following third molar surgery: a controlled, prospective study. J Ora Rehabil. 2009;36(3):199-209.

10. Sharma $U$, Narain $S$. Unusual facial pain secondary to inferior alveolar nerve compression caused by impacted mandibular second molar. J. Indian Soc Pedod Prev Dent. 2014;32(2):164.

11. Spencer HR, Morrison A, Braga A, Brennan PA. Review article: oral surgery and TMJ-related papers published in BJOMS in 2008 and 2009. Br. J. Ora Maxillofac Surg. 2010;48(7):544-8.

12. Kehlet $\mathrm{H}$, Jensen TS, Woolf CJ. Persistent postsurgical pain: risk factors and prevention. Lancet. 2006:367(9522):1618-25.

13. Perkins FM, Kehlet $\mathrm{H}$. Chronic pain as an outcome of surgery: A review of predictive factors. Anesthesiology. 2000;93(4):1123-33.

14. Dolwick MF, Widmer CG. Orthognathic Surgery as a Treatment for Temporomandibular Disorders. Oral Maxillofac Surg Clin North Am. 2018;30(3):303-23.

15. Pereira LJ, Pereira-Cenci T, Cury AADB, Pereira SM, Pereira AC, Ambosano $\mathrm{GMB}$, et al. Risk Indicators of Temporomandibular Disorder Incidences in Early Adolescence. Pediatr Dent. 2010;32(4):324-8. 